\title{
Multi-Step Finite Control Set Model Predictive Control For Three- level APF
}

\author{
DeJiYangZong ${ }^{1}$, Shifeng $\mathrm{Hu}^{1, *}$, XuGuang Yang ${ }^{1}$ \\ ${ }^{1}$ Electric Engineering College, Tibet Agriculture \& Animal Husbandry University 860000, China
}

\begin{abstract}
Compared with the two-level APF, the three-level neutral point clamped (ANPC) has more output levels, less harmonic influence, and lower equipment loss in the process of harmonic compensation. However, due to the conservatism of single-step prediction, it can only provide the reliability at $k+1$ time, and it does not provide the relevant information for the next time optimal. Moreover, the control of threelevel APF needs to consider multiple targets at the same time, which will cause certain tracking error. In this paper, a multi-step prediction method is proposed. The optimal and suboptimal switching states at $k+1$ are obtained by cycling. The optimal switching states in two control cycles can be obtained by substituting the optimal switching states at $k+2$. The method improves the reliability of the control process, and the simulation results show that the harmonic content of the power grid is low, which meets the relevant requirements.
\end{abstract}

\section{Introduction}

With the wide use of many power electronic equipment and the access of new energy generation to the power grid, the content of reactive power and harmonic in the power grid increases greatly, and the power quality is seriously reduced. However, the guarantee of power quality is the basis of all kinds of power equipment operation, so the improvement of power quality is an urgent problem to be solved, and APF is a widely used device to improve power quality, which has become a hot research direction. Its working principle is to inject the detected harmonic current into the power grid in the opposite direction. APF can not only reduce the harmonic content, but also compensate the reactive power, which can effectively improve the power quality and improve the power supply security of distribution network [1-2].

With the wide use of APF, many scholars have proposed various control methods for APF, and the reliability of harmonic compensation and governance for APF has been further improved. In reference [3], a repetitive control strategy for APF is proposed to improve the tracking of periodic current reference required by $\mathrm{APF}$, and the feasibility of the algorithm is verified by experiments; in reference [4], an optimal control strategy for NPC structure APF is proposed, a suitable quadratic weighed cost function is used to choose the voltage vector that minimizes the $\mathrm{AC}$ current tracking errors.

Model predictive control (MPC) is a kind of control technology which appears with the development of industry. It is suitable for multivariable and constrained systems. Later, it was gradually applied to power electronics, and the finite set model predictive control (FCS-MPC) was proposed to deal with the limited combination of switching devices. FCS-MPC has many advantages, such as fast response to sudden load change, ability to control multiple targets, clear physical meaning, simple algorithm, and easy implementation. In this paper, FCS-MPC is applied to APF with three-level NPC structure. Compared with two-level structure, it has more abundant vector combinations, better tracking effect and lower consumption. Aiming at the conservative type of one-step prediction, this paper studies the multi-step prediction strategy of FCS-MPC with three-level NPC structure [5], which predicts the optimal switching state in two cycles, increasing the reliability and conservatism. Finally, the effectiveness and feasibility of the control method are verified by experiments and simulation.

\section{The structure and mathematical model of three-level APF}

\subsection{Topology}

The main circuit structure of three-phase three wire APF is shown in Figure 1. There are 12 IGBTs, 12 freewheeling diodes and 6 clamping diodes. $C_{1}$ and $C_{2}$ are DC side balancing capacitors with equal capacitance values. $e_{a}, e_{b}, e_{c}$ are grid voltage, $i_{c a}, i_{c b}$ and $i_{c c}$ are output compensation current of APF, $i_{L a}, i_{L b}$, and $i_{L c}$ are load current, $L$ and $R$ are filter inductance and equivalent resistance.

\footnotetext{
*sushe11203@126.edu.cn
} 


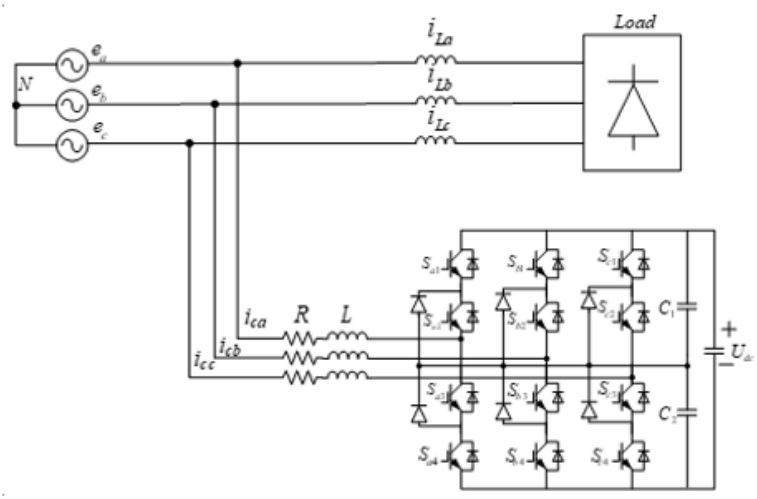

Fig.1 Structure diagram of three-level APF

For the three-level APF, each bridge arm has 4 points of IGBT, and there are 27 different switching states in total. From this, it can be defined that the formula of the switching function is equation (1).

$$
S_{x}= \begin{cases}P & S_{x 1}=S_{x 2}=1, S_{x 3}=S_{x 4}=0 \\ 0 & S_{x 1}=S_{x 4}=0, S_{x 2}=S_{x 3}=1 ; \\ N & S_{x 1}=S_{x 2}=0, S_{x 3}=S_{x 4}=1\end{cases}
$$

The output voltage at each end can be expressed as:

$$
u_{x o}=S_{x} * u_{d c} ; \quad(x=a, b, c)
$$

The 27 switching states of the three-level APF can generate 19 different voltage vectors, including three sets of zero vectors and 12 sets of redundant vectors. These 27 switching states and vectors can be expressed as Fig. 2

\subsection{Mathematical model of three-level APF}

According to the structure diagram of Fig. 1, the mathematical model of $a, b$ and $c$ three-phase can be obtained as equation (2), where $u_{c a}$ is the output voltage value of $\mathrm{APF}$.

$$
\left\{\begin{array}{l}
L \frac{\mathrm{d} i_{a}}{\mathrm{~d} t}=R i_{a}+e_{a}+u_{c a} \\
L \frac{\mathrm{d} i_{b}}{\mathrm{~d} t}=R i_{b}+e_{b}+u_{c b} \\
L \frac{\mathrm{d} i_{c}}{\mathrm{~d} t}=R i_{c}+e_{c}+u_{c c}
\end{array}\right.
$$

Since in the three-phase coordinate system, the current output tracking reference value will have cumulative errors, the mathematical model is established in the $\alpha-\beta$ coordinate system.

$$
\left\{\begin{array}{l}
L \frac{\mathrm{d} i_{\alpha}}{\mathrm{d} t}=u_{c \alpha}-e_{\alpha}-R i_{\alpha} \\
L \frac{\mathrm{d} i_{\beta}}{\mathrm{d} t}=u_{c \beta}-e_{\beta}-R i_{\beta}
\end{array}\right.
$$

The dynamic equation of DC side capacitor $C_{1}, C_{2}$ is equation (4)

$$
\begin{aligned}
\frac{\mathrm{d} u_{c 1}}{\mathrm{~d} t} & =\frac{1}{C} i_{c 1} \\
\frac{\mathrm{d} u_{c 2}}{\mathrm{~d} t} & =\frac{1}{C} i_{c 2}
\end{aligned}
$$

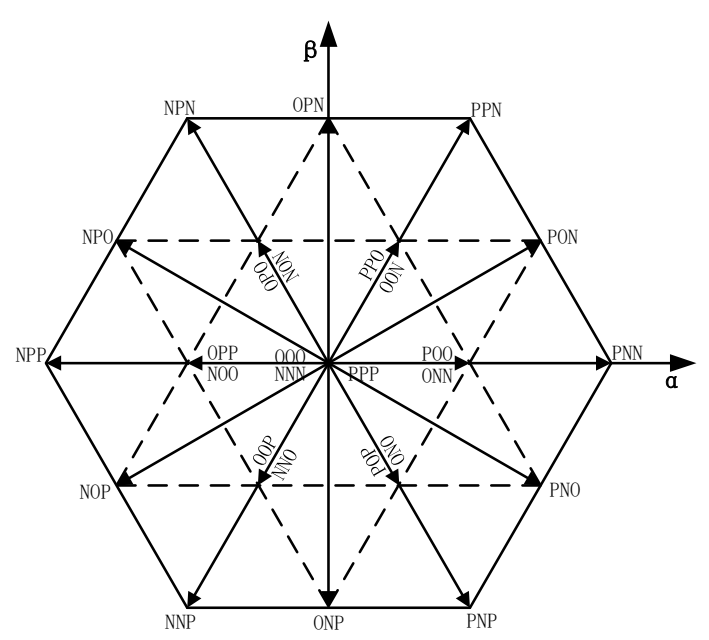

Fig.2 27 sets of switches vector

\section{Predictive Control of Traditional Three-level APF}

\subsection{FCS-MPC principle of current}

In the traditional model predictive control technology of three-level APF, the reference current is calculated by discrete mathematical model, and then 27 vectors are optimized to find the switch combination closest to the reference current vector as the output at $k+1$. At the same time, in order to minimize the objective function, the problem of neutral point voltage balance should be considered at the same time. In the $\alpha-\beta$ coordinate system, the action state of APF at $\mathrm{k}$ time has been obtained from the previous time, and then 27 voltage vectors are cycled to select the switching state which makes the objective function minimum to act on APF at $k+1$. The schematic diagram of predictive control can be obtained, as shown in Figure 3. At each sampling time, all switching states are cycled in turn, and the detected harmonic current value is taken as the reference value, and the variance of the predicted value is calculated. At the same time, the term to balance the capacitor voltage is added to distribute the weight factor, synthesize all the reference tracking values, select the switching state to minimize the value function, and output it to APF. In this way, the harmonic current can be tracked in real time without pulse width modulation (PWM). 


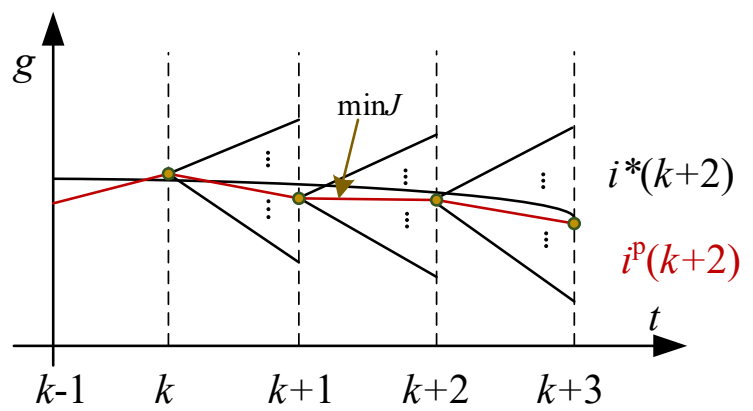

Fig.3 Diagram of FCS-MPC principle

\subsection{Establishment of Prediction Model and Objective Function}

After discretizing the difference of equations (4) and (5), equations (6) and (7) are obtained

$$
\left\{\begin{array}{l}
i_{\alpha}(k+1)=\frac{T_{s}}{L} u_{c \alpha}(k)-\frac{T_{s}}{L} e_{\alpha}(k)+\left(1-\frac{R T_{s}}{L}\right) i_{\alpha}(k) \\
i_{\beta}(k+1)=\frac{T_{s}}{L} u_{c \beta}(k)-\frac{T_{s}}{L} e_{\beta}(k)+\left(1-\frac{R T_{s}}{L}\right) i_{\beta}(k)
\end{array}\right.
$$

Since the reference harmonic current is at the current moment, the second-order LaGrange interpolation prediction is performed on it

$$
i^{*}(k+1)=4 i^{*}(k)-6 i^{*}(k-1)+4 i^{*}(k-2)-i^{*}(k-3)
$$

According to the above prediction equation, the cost function of equation (11) can be established

$$
J=\lambda_{1}\left\|i_{\alpha}^{*}(k+1)-i_{\alpha}(k+1)\right\|+\lambda_{2}\left\|i_{\beta}^{*}(k+1)-i_{\beta}(k+1)\right\|+\lambda_{3}\left\|u_{c 1}(k+1)-u_{c 2}(k+1)\right\|
$$

\section{$4 \quad$ Multistep FCS-MPC with Three-level NPC Structure for APF}

The traditional control method can achieve the optimal solution in one control cycle, but it cannot achieve the optimal solution in two or more cycles in the future. Although the single step algorithm has low computation and low structure, it is conservative to some extent, that is, the switch state obtained is only the optimal solution at $k+1$ time, without considering the optimality at $k+2$ and $k+3$ time, and missing the optimal information that other switch states may contain. If there is error or interference in the system, its conservatism will affect the control performance. In the following multiple control cycles, 17 groups of switch states cannot find the appropriate state, which makes the predicted current closer to the reference current, so that the system has greater error and reduces the filtering effect.

Multi step FCS-MPC selects the switch state at this time, synthesizes the optimal objective function in two cycles, and selects the optimal switch state to act on APF.

a) At $k$ time, according to the current value $i(k)$, the reference current at the next time is obtained by Lagrange interpolation.

b) The objective function is constructed, 27 groups of switch combinations are cycled, and the switch combinations which make the objective function minimum and the second smallest are selected. The two groups of switch states which make the objective function minimum are calculated respectively, and the optimal current prediction values $\mathrm{i}_{1}^{\mathrm{p}}(k+1)$ and $\mathrm{i}_{2}^{\mathrm{p}}(k+1)$ are obtained, and the corresponding switching states are recorded.

c) Using the two groups of predicted values corresponding to the objective function at $k+1$ obtained in the second part, 27 groups of vectors are recycled again to display the switch state corresponding to the minimum objective function at $k+2$. The same method is applied to the current prediction value $\mathrm{i}^{\mathrm{p}}(k+2)$ to obtain two objective functions at $k+2$.

d) The objective function at $k+1$ time and the smaller one at $k+2$ time are selected as the final prediction results, and the corresponding switch state at $k+1$ time is taken as the application state at $k+1$ time and applied to APF.

From the above analysis, the advantage of the multistep fcs-mpc proposed in this paper over the traditional model prediction algorithm is that it seeks the optimal solution at the current time and in the subsequent multiple cycles by solving the objective function through multiple rolling optimization algorithm, so as to solve the conservative problem of the traditional algorithm. Because the computational complexity is increased by 
three times, the performance of the processor is sacrificed in exchange for the optimal switch combination. The better the processor performance, the more obvious the control performance will be.

\section{Simulation Analysis}

In order to verify the effectiveness of the multi-step FCS-MPC control strategy for the three-level APF, a simulation model was built in MATLAB/Simulink, and the parameters are shown in Table 1. The harmonic source is an uncontrollable diode rectifier bridge, and the load fluctuates at 0.3 seconds. The reference current is the harmonics detected by the ip-iq method. Take $\lambda_{1}=\lambda_{2}=0.45$ and $\lambda_{3}=0.1$.

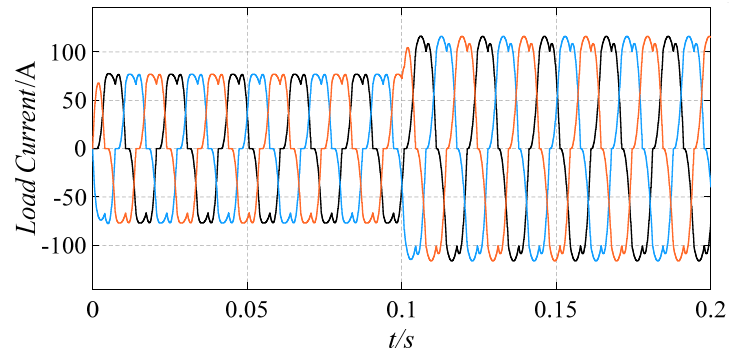

(a)

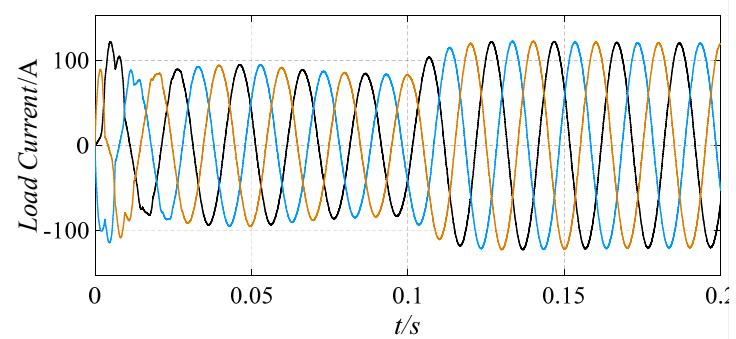

(c)

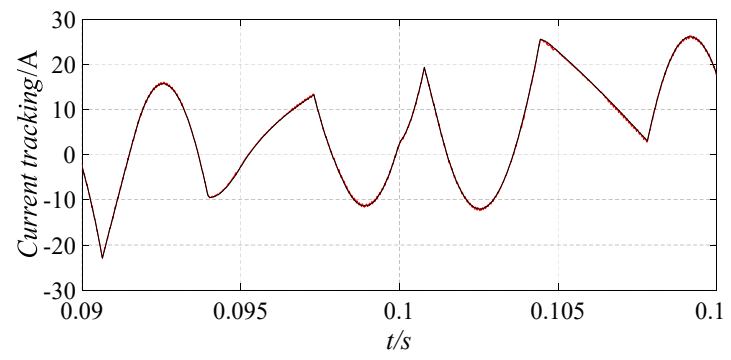

(e)
Table.1 Simulation model parameters

\begin{tabular}{ll}
\hline Parameters & Value \\
\hline Grid Voltage & $\mathbf{3 8 0 V}$ \\
DC Voltage & $\mathbf{8 0 0 V}$ \\
Capacitor $C_{1}, C_{2}$ & $\mathbf{3 0 0 0 \mu \mathbf { F }}$ \\
Inductor & $\mathbf{4 m H}$ \\
Resistor & $\mathbf{0 . 0 1 \Omega}$ \\
Load Resistor & $\mathbf{1 0 \Omega}$
\end{tabular}

Setting the above parameters of the simulation model, and running the simulation in Simulink, you can get the simulation diagrams in Fig. 4, which can reflect the dynamic performance and stability of the algorithm.

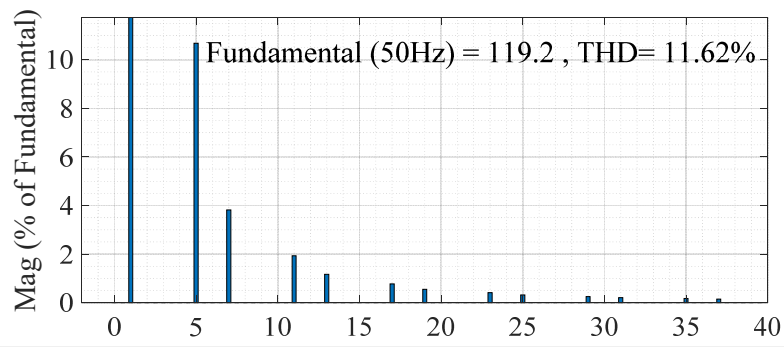

(b)

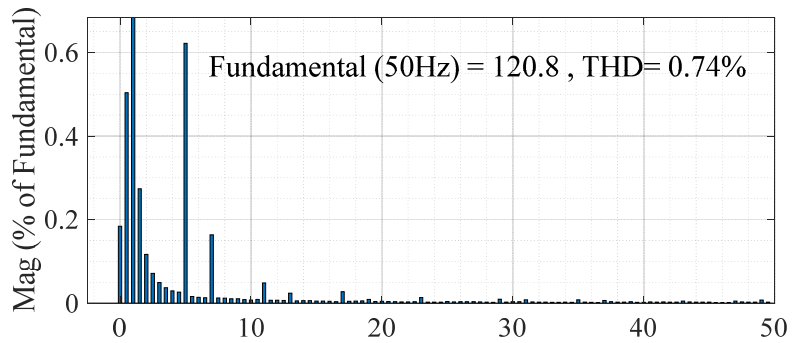

(d)

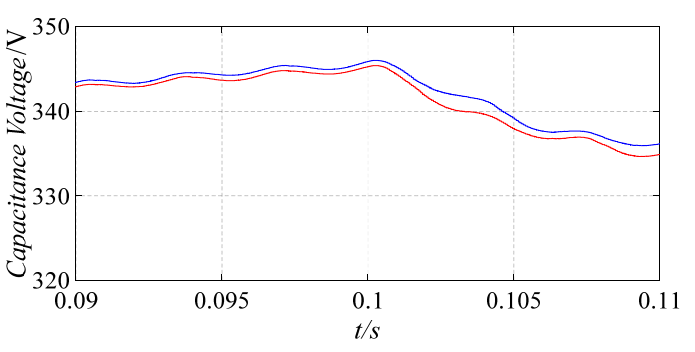

(f)

Fig.4 (a) is the three-phase load current before compensation; (b) is the THD; (c) is the grid current after compensation;(d) is the THD: (e) is the effect picture of reference current tracking; (f) is the voltage value of capacitors $C_{1}$ and $C_{2}$;

The (a) is the three-phase harmonic load current, it can be seen from (b) that through $i_{p}-i_{q}$ method, the harmonics of power grid are mainly 5 th, 7 th, 11 th and 13th harmonics, and the harmonic content is higher. (c) In order to apply multi-step FCS-MPC algorithm to control APF for compensation, it can be seen from (d) that $\mathrm{THD}=0.74 \%$, and the algorithm has good compensation effect. (c) In order to compensate and track the harmonic reference current, the APF output can follow the reference current stably. (d) For the balance effect of the two capacitors, under the influence of the objective function, the voltage of the two capacitors can be balanced stably.

\section{Conclusion}

Aiming at the conservatism of one-step FCS-MPC algorithm, this paper proposes a multi-step predictive control method. Based on the three-level APF mathematical model of NPC structure, the objective 
function is established to track the reference harmonic and balance the neutral point voltage. The simulation results show that the control accuracy of the algorithm is improved, the effect of harmonic compensation is improved, and the fast response ability of load mutation is verified.

\section{Acknowledgement}

Natural Science Foundation of Tibet Autonomous Region (XZ202001ZR0093G)

\section{References}

1. B. Singh, K. Al-Haddad and A. Chandra, "A review of active filters for power quality improvement," in IEEE Transactions on Industrial Electronics, vol. 46, no. 5, pp. 960-971, Oct. 1999, doi: 10.1109/41.793345.

2. O. Vodyakho and C. C. Mi, "Three-Level InverterBased Shunt Active Power Filter in Three-Phase Three-Wire and Four-Wire Systems," in IEEE Transactions on Power Electronics, vol. 24, no. 5, pp. 1350-1363, May 2009, doi: 10.1109/TPEL.2009.2016663.

3. A. Garcia-Cerrada, O. Pinzon-Ardila, V. FeliuBatlle, P. Roncero-Sanchez and P. Garcia-Gonzalez, "Application of a Repetitive Controller for a ThreePhase Active Power Filter," in IEEE Transactions on Power Electronics, vol. 22, no. 1, pp. 237-246, Jan. 2007, doi: 10.1109/TPEL.2006.886609.

4. J. D. Barros and J. F. Silva, "Optimal Predictive Control of Three-Phase NPC Multilevel Converter for Power Quality Applications," in IEEE Transactions on Industrial Electronics, vol. 55, no. 10, pp. 3670-3681, Oct. 2008, doi: 10.1109/TIE.2008.928156.

5. A. Nabae, I. Takahashi and H. Akagi, "A New Neutral-Point-Clamped PWM Inverter," in IEEE Transactions on Industry Applications, vol. IA-17, no. 5, pp. 518-523, Sept. 1981, doi: 10.1109/TIA.1981.4503992.

6. A. Fahmy, M. S. Hamad, A. K. Abdelsalam and A. Lotfy, "Power quality improvement in three-phase four-wire system using a shunt APF with predictive current control," IECON 2012 - 38th Annual Conference on IEEE Industrial Electronics Society, Montreal, QC, 2012, pp. 668-673, doi: 10.1109/IECON.2012.6388748. 